\title{
On the Convergence of a Crank-Nicolson Fitted Finite Volume Method for Pricing American Bond Options
}

\author{
Xiaoting Gan $\mathbb{D}^{1,2}$ and Dengguo $\mathrm{Xu} \mathbb{D}^{3}$ \\ ${ }^{1}$ School of Mathematics and Statistics, Chuxiong Normal University, Chuxiong, Yunnan 675000, China \\ ${ }^{2}$ School of Mathematical Sciences, University of Electronic Science and Technology of China, Chengdu 611731, China \\ ${ }^{3}$ School of Physics and Electrical Engineering, Liupanshui Normal University, Liupanshui, Guizhou 553004, China \\ Correspondence should be addressed to Xiaoting Gan; 9xtgan@alumni.tongji.edu.cn
}

Received 27 March 2020; Revised 29 April 2020; Accepted 6 May 2020; Published 28 May 2020

Academic Editor: Dimitrios Mitsotakis

Copyright (c) 2020 Xiaoting Gan and Dengguo Xu. This is an open access article distributed under the Creative Commons Attribution License, which permits unrestricted use, distribution, and reproduction in any medium, provided the original work is properly cited.

\begin{abstract}
This paper develops and analyses a Crank-Nicolson fitted finite volume method to price American options on a zero-coupon bond under the Cox-Ingersoll-Ross (CIR) model governed by a partial differential complementarity problem (PDCP). Based on a penalty approach, the PDCP results in a nonlinear partial differential equation (PDE). We then apply a fitted finite volume method for the spatial discretization along with a Crank-Nicolson time-stepping scheme for the PDE, which results in a nonlinear algebraic equation. We show that this scheme is consistent, stable, and monotone, and hence, the convergence of the numerical solution to the viscosity solution of the continuous problem is guaranteed. To solve the system of nonlinear equations effectively, an iterative algorithm is established and its convergence is proved. Numerical experiments are presented to demonstrate the accuracy, efficiency, and robustness of the new numerical method.
\end{abstract}

\section{Introduction}

Pricing and hedging of interest rate derivatives, such as bond options, have been increasingly attracting much attention by mathematicians and financial engineers in the last two decades [1-3]. Usually, bond option has a bond as its underlying asset whose price depends on both interest rate and time. Compared to stock derivatives, the valuation of these types of options is more complicated and poses greater challenges. In this paper, we focus on pricing American options on a zero-coupon bond under the CIR model governed by a PDCP (1). Due to the early exercise feature, this PDCP is, in general, not analytically solvable, and then, the efficient numerical approximation methods are normally required [4].

In the past few years, the numerical solution of the American bond option pricing problem has been investigated by various approximation techniques, e.g., the lattice method, the finite difference method, the finite element method, and the fitted finite volume method. For more details, we refer the readers to [5-14] and references therein. However, it should be noted that due to the degeneracy of the nonlinear PDE, many research studies, e.g., $[5,11,15]$, have been pointed out that most of the above methods are difficult to solve the PDE accurately and/or only convergent for certain combinations of parameters. Recently, the fitted finite volume method for pricing stock options governed by standard Black-Scholes equations has attracted much attention. This method was first used to price European stock options by Wang [16] and then generalized to other types of options by many researchers (see, e.g., [14, 17-21] and references therein). Actually, the fitted finite volume method is based on a finite volume formulation (cf. [22, 23]) coupled with a popular exponentially fitting approximation technique. It has been shown that this method has greater success in pricing stock options and is gaining popularity because it can overcome the difficulty caused by driftdominated phenomena very well. Besides, it is well known that based on the penalty approach, the nonlinear penalized PDEs resulting from American options models are usually 
degenerated and drift-dominated, and hence, the fitted finite volume method is a natural way to overcome these difficulties [17].

The fitted finite volume method in $[11,14]$ evaluates the American and European options on a zero-coupon bond under the CIR model, respectively, and the corresponding PDEs are treated by a fully implicit (i.e., backward Euler) time-stepping scheme. We note that the scheme has the firstorder convergence rate. To the authors' knowledge, based on the fitted finite volume method discretization, there is no theoretical result published about the Crank-Nicolson timestepping scheme which will provide the second order of convergence to price this problem for the time being. On this basis, in this paper, we derive a novel fitted finite volume method combined with the Crank-Nicolson scheme for pricing American options on a zero-coupon bond under the CIR model. Based on a penalty approach, the PDCP (1) results in a nonlinear penalized PDE, and then, we apply the fitted finite volume method in space, coupled with the Crank-Nicolson scheme in time. We show that this numerical scheme is consistent, stable, and monotone, and hence, the convergence of the numerical solution to the viscosity solution of the continuous problem is guaranteed. Further, to solve the nonlinear system effectively, we design an iterative method and prove that the method is convergent. Finally, some numerical experiments are implemented to verify the accuracy, efficiency, and robustness of our numerical method. The numerical results further show that the Crank-Nicolson fitted finite volume scheme provides the rate of convergence is of approximately second order.

The paper is organized as follows: in the next section, we introduce American options on a zero-coupon bond model and give a penalty approach to the PDCP. Then, a Crank-Nicolson fitted finite volume scheme is constructed in Section 3. In Section 4, the convergence of the numerical scheme is investigated. In Section 5, we propose an iterative algorithm for the nonlinear system and establish a convergence theory. Numerical tests are performed in Section 6. The paper ends with some conclusions.

\section{Mathematical Model and the Penalty Approach}

In this work, we assume the short-term interest rate (denoted by " $r$ ") structure is governed by the CIR model, i.e., $r$ is governed by the following mean-reverting version of the square-root process:

$$
\mathrm{d} r=\kappa(\theta-r) \mathrm{d} t+\sigma \sqrt{r} \mathrm{~d} W,
$$

where $d W$ is the increment of a Wiener process, $\theta$ is the long-term level of the short rate, $\kappa>0$ stands for the reversion speed, and $\sigma^{2} r$ is the variance with $\sigma>0$. In [24], it has been shown that the price $P(r, t, s)$ of a pure discount bond with face value $\$ 1$ at its maturity date $s$ is given as follows:

$$
P(r, t, s)=\alpha(t, s) e^{-\beta(t, s) r},
$$

where

$$
\begin{aligned}
\alpha(t, s) & =\left[\frac{\phi_{1} e^{\phi_{2}(s-t)}}{\phi_{2}\left[e^{\phi_{1}(s-t)}-1\right]+\phi_{1}}\right]^{\phi_{3}}, \\
\beta(t, s) & =\frac{e^{\phi_{1}(s-t)}-1}{\phi_{2}\left[e^{\phi_{1}(s-t)}-1\right]+\phi_{1}}, \\
\phi_{1} & =\sqrt{\mu^{2}+2 \sigma^{2}}, \\
\phi_{2} & =\frac{\left(\mu+\phi_{1}\right)}{2}, \\
\phi_{3} & =\frac{2 \bar{\theta}}{\sigma^{2}}, \quad \bar{\theta}=\kappa \theta, \mu=\kappa+\zeta,
\end{aligned}
$$

and $\zeta$ is the market risk premium. At the maturity date $s$, the price of a pure discount bond is its face value, i.e.,

$$
P(r, t=s, s)=1 .
$$

Now, let $V(r, t)$ be the value of an American put option on a zero-coupon bond with striking price $K$, where the holder can receive a given payoff $\Lambda(r, t)$ at the expiry date $T$. Introducing a time-reverse transformation $\tau=T-t$, then the value $V(r, \tau)$ can be governed by the following PDCP (cf. [12]):

$$
\left\{\begin{array}{l}
L V(r, \tau) \geqslant 0 \\
V(r, \tau)-\Lambda(r, \tau) \geqslant 0 \\
L V(r, \tau)(V(r, \tau)-\Lambda(r, \tau))=0,
\end{array}\right.
$$

a.e. in $(0,+\infty) \times(0, T)$, where

$$
L V=\frac{\partial V}{\partial \tau}-\frac{1}{2} \sigma^{2} r \frac{\partial^{2} V}{\partial r^{2}}-(\bar{\theta}-\mu r) \frac{\partial V}{\partial r}+r V
$$

and the initial condition is given by

$$
V(r, 0)=\Lambda(r, 0)=\max \{K-P(r, T, s), 0\},
$$

and the boundary conditions are as follows:

$$
\begin{aligned}
& V(0, \tau)=\Lambda(r, \tau), r \longrightarrow 0 \\
& V(r, \tau)=\Lambda(r, \tau), r \longrightarrow \infty
\end{aligned}
$$

For computational purpose, it is necessary to restrict the short-term interest rate $r$ in a finite region $I=\left[0, r_{\max }\right]$, where $r_{\max }$ is a sufficiently large number to ensure the accuracy. Thus, the boundary conditions ( 8 ) become

$$
\begin{gathered}
V(0, \tau)=\Lambda(0, \tau), \\
V\left(r_{\max }, \tau\right)=\Lambda\left(r_{\max }, \tau\right) .
\end{gathered}
$$

By using the penalty method (cf. $[11,20,25]$ ), we can transform the PDCP (1) into the following nonlinear penalized PDE:

$$
\mathscr{L} V^{\gamma}(S, \tau)-\gamma\left[\Lambda-V^{\gamma}\right]_{+}=0,
$$

with the given initial and boundary conditions: 


$$
\left\{\begin{array}{l}
V^{\gamma}(r, 0)=\Lambda(r, 0), \\
V^{\gamma}(0, \tau)=K, \\
V^{\gamma}(R, \tau)=0,
\end{array}\right.
$$

where $\gamma$ is the penalty parameter satisfying $\gamma>1$ and $[x]_{+}=$ $\max \{0, x\}$ for any $x$. It is well known that, by adding the penalty term, the above penalty approach is to force the positive parts $\left[\Lambda-V^{\gamma}\right]_{+}$in (10) to be close to zero as $\gamma$ becomes sufficiently large. Hence, the complementarity condition in (5) is approximately satisfied.

Remark 1. Since the degeneracy of the diffusion operators $\mathscr{L}$, the nonsmoothness of payoff functions, and nonlinearity of $\max \{\cdot, \cdot\}$, both problems (5) and (10) have no classical solutions, in general. Therefore, we seek viscosity solutions to the PDCP (1) and penalized PDE (5). Detailed discussions concerning the existence and uniqueness of viscosity solutions to PDE in the context of financial mathematics are given in $[26,27]$, which are defined as follows.

Definition 1 (continuous viscosity solutions [27]). $u \in C(\Omega)$ is a viscosity solution of

$$
H\left(x, u, D u \quad, D^{2} u\right)=0 \quad \text { in } \Omega,
$$

where $H$ is a continuous function satisfying the ellipticity condition:

$$
H(x, u, p, M) \leq H(x, u, p, N) \quad \text { if } M \geq N,
$$

for any $x \in \Omega, \Omega$ is a domain in $R^{N}, u \in R, p \in R^{N}$, if and only if $\forall \varphi \in C^{2}(\Omega)$, if $x_{0} \in \Omega$ is a local maximum point of $u-\varphi$, one has

$$
H\left(x_{0}, u\left(x_{0}\right), D \varphi\left(x_{0}\right), D^{2} \varphi\left(x_{0}\right)\right) \leq 0,
$$

and $\forall \varphi \in C^{2}(\Omega)$, if $x_{0} \in \Omega$ is a local minimum point of $u-\varphi$, one has

$$
H\left(x_{0}, u\left(x_{0}\right), D \varphi\left(x_{0}\right), D^{2} \varphi\left(x_{0}\right)\right) \geq 0 .
$$

We note that, according to [11], the solution $V^{\gamma}$ to penalized PDE (5) is an approximation to solution $V$ of PDCP (1) in the viscosity sense. The convergence of $V^{\gamma}$ to $V$ as $\gamma \longrightarrow \infty$ has been discussed by many researchers, e.g., Li and Wang [28] and Wang et al. [29]. Therefore, we will concentrate on the numerical approximation of the viscosity solution to PDE (5). Besides, to avoid an overloading of symbols, we omit the subscript $\gamma$ of $V^{\gamma}$ used in the above, but bear in mind that this $V$ is the solution of penalized PDE (5).

\section{The Crank-Nicolson Fitted Finite Volume Method}

In this section, we will present a Crank-Nicolson fitted finite volume method for the nonlinear penalized PDE (5).

3.1. Semidiscretization Scheme. Before proceeding to the Crank-Nicolson fitted finite volume discretization scheme, we first transform PDE (5) into the following conservative form:

$$
\frac{\partial V}{\partial \tau}=\frac{\partial}{\partial r}\left[\operatorname{ar} \frac{\partial V}{\partial S}+b(r) V\right]-c(r) V+\gamma[\Lambda-V]_{+},
$$

where

$$
\left\{\begin{array}{l}
a=\frac{\sigma^{2}}{2}>0, \\
b(r)=\bar{\theta}-\mu r-\frac{\sigma^{2}}{2}, \\
c(r)=r-\mu .
\end{array}\right.
$$

The fitted finite volume method is based on the selfadjoint form (16). Now we first define two spatial partitions of $I=\left[0, r_{\max }\right]$. Let $I$ be divided into $M$ subintervals:

$$
I_{i}=\left(r_{i}, r_{i+1}\right), \quad i=0,1, \ldots, M-1,
$$

with $0=r_{0}<r_{1}<r_{2}<\cdots<r_{M}=r_{\max }$. For each $i=0,1, \ldots, M-1$, let $h_{i}=r_{i+1}-r_{i}$. Also, we let $r_{i-1 / 2}=\left(r_{i-1}+r_{i}\right) / 2$ and $r_{i+1 / 2}=\left(r_{i}+r_{i+1}\right) / 2$ for each $i=1, \ldots, M-1$. Then these intervals $I_{i}^{*}=\left(r_{i-1 / 2}, r_{i+1 / 2}\right)$, $i=0,1, \ldots, M$, form a second partition of $I=\left[0, r_{\max }\right]$ if we define $r_{-1 / 2}=r_{0}$ and $r_{M+1 / 2}=r_{M}$.

For each $i=1, \ldots, M-1$, integrating (16) over $I_{i}^{*}$, we have

$$
\begin{aligned}
\int_{I_{i}^{*}} \frac{\partial V}{\partial \tau} \mathrm{d} r= & {\left[a r \frac{\partial V}{\partial r}+b(r) V\right]_{r_{i-1 / 2}}^{r_{i+1 / 2}}-\int_{I_{i}^{*}} c(r) V \mathrm{~d} r } \\
& +\gamma \int_{I_{i}^{*}}[\Lambda-V]_{+} \mathrm{d} r .
\end{aligned}
$$

Applying the one-point quadrature rule to all the terms in (19) except the first term in the right-hand side, we obtain

$$
\begin{aligned}
\frac{\partial V_{i}}{\partial \tau} l_{i}= & \left.\rho(V)\right|_{r_{i+1 / 2}}-\left.\rho(V)\right|_{r_{i-1 / 2}}-c_{i} l_{i} V_{i} \\
& +\gamma l_{i}\left[\Lambda_{i}-V_{i}\right]_{+}, \quad i=1, \ldots, M-1,
\end{aligned}
$$

where $l_{i}=r_{i+1 / 2}-r_{i-1 / 2}$ is the length of interval $I_{i}^{*}$ and $\rho(V)$ is the weighted flux density associated with $V$ defined as follows:

$$
\rho(V):=a r V^{\prime}+b(r) V,
$$

where $V_{i}$ and $\Lambda_{i}$ denote the approximation of $V\left(r_{i}, \tau\right)$ and $\Lambda\left(r_{i}, \tau\right)$, respectively.

As the discretization of (20) and (21) is identical to that in $[11,14]$, so we omit the details and only give the final discrete form here, i.e., the semidiscretization of the PDE (6) is given by

$$
\frac{\partial \mathbf{V}}{\partial \tau}=\mathbf{S V}+\mathbf{P}(\Lambda-\mathbf{V})+\mathbf{F},
$$

where $\mathbf{V}=\left[V_{1}, V_{2}, \ldots, V_{M-1}\right]^{T}, \quad \Lambda=\left[\Lambda_{1}, \Lambda_{2}, \ldots, \Lambda_{M-1}\right]^{T}$, and $\mathbf{P}$ is a $(M-1) \times(M-1)$ diagonal matrix with 


$$
P_{i i}= \begin{cases}\gamma, & \text { if } V_{i}<\Lambda_{i}, \\ 0, & \text { otherwise, }\end{cases}
$$

and $\mathbf{S}$ is a $(M-1) \times(M-1)$ semidiscretization matrix given by

$$
\mathbf{S}=\left(\begin{array}{cccc}
\chi_{1} & w_{1} & & \\
\beta_{2} & \chi_{2} & w_{2} & \\
& \ddots & \ddots & \ddots \\
& & \beta_{M-1} & \chi_{M-1}
\end{array}\right)_{(M-1) \times(M-1)}
$$

where

$$
\begin{aligned}
& \beta_{1}=\frac{1}{2 l_{1}}\left(a-b_{1 / 2}\right), \\
& w_{1}=\frac{b_{3 / 2} r_{2}^{\eta_{1}}}{\left(r_{2}^{\eta_{1}}-r_{1}^{\eta_{1}}\right) l_{1}}, \\
& \chi_{1}=-\frac{1}{2 l_{1}}\left(a+b_{1 / 2}\right)-\frac{b_{3 / 2} r_{1}^{\eta_{1}}}{\left(r_{2}^{\eta_{1}}-r_{1}^{\eta_{1}}\right) l_{1}}-c_{1}, \\
& \beta_{i}=\frac{b_{i-1 / 2} r_{i-1}^{\eta_{i-1}}}{\left(r_{i}^{\eta_{i-1}}-r_{i-1}^{\eta_{i-1}}\right) l_{i}}, \\
& \chi_{i}=-\frac{b_{i-1 / 2} r_{i}^{\eta_{i-1}}}{\left(r_{i}^{\eta_{i-1}}-r_{i-1}^{\eta_{i-1}}\right) l_{i}}-\frac{b_{i+1 / 2} r_{i}^{\eta_{i}}}{\left(r_{i+1}^{\eta_{i}}-r_{i}^{\eta_{i}}\right) l_{i}}-c_{i}, \\
& w_{i}=\frac{b_{i+1 / 2} r_{i+1}^{\eta_{i}}}{\left(r_{i+1}^{\eta_{i}}-r_{i}^{\eta_{i}}\right) l_{i}},
\end{aligned}
$$

for $i=2, \ldots, M-1, \mathbf{F}=\left[\beta_{1} V_{0}, 0, \ldots, 0, w_{M-1} V_{M}\right]^{T}$, and $V_{0}$ and $V_{M}$ being equal to the given boundary conditions in (9), and $\eta_{i}=b_{i+1 / 2} / a$.

3.2. The Crank-Nicolson Scheme. We now consider the timestepping scheme to (22). Let $\tau_{n}=n \Delta \tau(n=0,1, \ldots, N)$ be a set of partition points in $[0, T]$ with subinterval length $\Delta \tau=T / N$, where $N$ is a positive integer. It has been shown in $[11,14]$ that the fully implicit fitted finite volume scheme is only first-order accurate for pricing both European and American zero-coupon bond options under the CIR model. To improve the accuracy in time step, the second-order Crank-Nicholson scheme should be employed here. Thus, the Crank-Nicolson scheme of (22) can be written in the following matrix form:

$$
\begin{aligned}
{\left[\mathbf{I}-\frac{\Delta \tau}{2} \mathbf{S}+\Delta \tau \mathbf{P}\left(\mathbf{V}^{n+1}\right)\right] \mathbf{V}^{n+1}=} & \left(\mathbf{I}+\frac{\Delta \tau}{2} \mathbf{S}\right) \mathbf{V}^{n} \\
& +\Delta \tau \mathbf{P}\left(\mathbf{V}^{n+1}\right) \Lambda+\Delta \tau \mathbf{F}^{n}
\end{aligned}
$$

for $n=0,1, \ldots, N-1$, where $\mathbf{V}^{n}=\left[V_{1}^{n}, V_{2}^{n}, \ldots, V_{M-1}^{n}\right]^{T}$, $\mathbf{F}^{n}=\left[\beta_{1} V_{0}^{n}, 0, \ldots, 0, w_{M-1} V_{M}^{n}\right]^{T}$, and $\mathbf{P}\left(\mathbf{V}^{n+1}\right)$ is a $(M-1) \times(M-1)$ diagonal matrix with

$$
\mathbf{P}\left(\mathbf{V}^{n+1}\right)_{i i}= \begin{cases}\gamma, & \text { if } V_{i}^{n+1}<\Lambda_{i}, \\ 0, & \text { otherwise }\end{cases}
$$

where $\mathbf{I}$ is a $(M-1) \times(M-1)$ unit matrix and $V_{i}^{n}$ denotes the approximations of $V\left(r_{i}, \tau_{n}\right)$ at $\tau=\tau_{n}$ for $i=1,2, \ldots, M-1$.

We comment that in (26), the Dirichlet boundary conditions (9) have been incorporated, and the initial condition is also incorporated as the payoff function. Furthermore, from [11], we can know that the matrix $-\mathbf{S}$ is an $M$-matrix. So, combing the definition of $\mathbf{P}\left(\mathbf{V}^{n+1}\right)$, we further have the following result for the numerical scheme (26).

Theorem 1. Both matrices $\mathbf{I}-(\Delta \tau / 2) \mathbf{S}$ and $\mathbf{I}-(\Delta \tau / 2) \mathbf{S}+$ $\Delta \tau \mathbf{P}\left(\mathbf{V}^{n+1}\right)$ are $M$-matrices.

Remark 2. A real $n \times n$ matrix $\mathbf{A}=\left[a_{i j}\right]$ with $a_{i j} \leq 0$ for all $i \neq j$ is an $M$-matrix if $\mathbf{A}$ is nonsingular and $\mathbf{A}^{-1} \geq O$, and further if $\mathbf{A}=\left[a_{i j}\right]$ is a real, irreducibly diagonally dominant $n \times n$ matrix with $a_{i j} \leq 0$ for all $i \neq j$, and $a_{i i}>0$ for all $1 \leq i \leq n$, then $\mathbf{A}^{-1} \geq O$ (cf. Varga [30], p. 91).

Remark 3. Theorem 1 implies that the Crank-Nicolson scheme (26) satisfies the discrete maximum principle, which guarantees that the discrete arbitrage inequality holds in option pricing theory.

\section{Convergence of the Numerical Scheme}

In this section, we investigate the convergence property of numerical scheme (26). First, the following lemma shows that scheme (26) is consistent.

Lemma 1. The numerical scheme (26) is consistent.

Proof. From the fitted finite volume discretization, we can see that the consistency of the numerical scheme (26) relies on the consistency of $\rho(V)$. Let $u$ be a sufficiently smooth function and $u_{h}$ be the discrete approximation of $u$. It has been shown in $[11,16]$ that the exact and the discrete flux yield

$$
\left|\left[\rho(u)-\rho_{h}\left(u_{h}\right)\right]_{r_{i+1 / 2}}\right|=O(h),
$$

where $\rho_{h}(V)$ satisfies

$$
\rho_{h}(V)=\rho_{i}(V), \quad \forall r \in I_{i},
$$

for $i=0,1, \ldots, M-1$, and $h=\max \left\{\max _{i} h_{i}, \Delta \tau\right\}$ be the mesh parameter. Hence, it is easy to see that the consistency of the numerical scheme (26) is the consequent result.

The stability result for the numerical scheme (11) is given as follows.

Lemma 2. The numerical scheme (26) is stable as $h \longrightarrow 0$, i.e.,

$$
\left\|\mathbf{V}^{n}\right\|_{\infty} \leq\|\Lambda\|_{\infty} .
$$


Proof. Writing out (26) in component form gives

$$
\begin{aligned}
{[1} & \left.-\frac{\Delta \tau}{2} \chi_{i}+\Delta \tau P\left(V^{n+1}\right)_{i i}\right] V_{i}^{n+1}-\frac{\Delta \tau}{2} \beta_{i} V_{i-1}^{n+1}-\frac{\Delta \tau}{2} w_{i} V_{i+1}^{n+1} \\
& =\left(1+\frac{\Delta \tau}{2} \chi_{i}\right) V_{i}^{n}+\frac{\Delta \tau}{2} \beta_{i} V_{i-1}^{n}+\frac{\Delta \tau}{2} w_{i} V_{i+1}^{n} \\
& +\Delta \tau P\left(V^{n+1}\right)_{i i} \Lambda_{i}+\Delta \tau F_{i}^{n} .
\end{aligned}
$$

$$
\begin{aligned}
& {\left[1-\frac{\Delta \tau}{2} \chi_{i}+\Delta \tau P\left(V^{n+1}\right)_{i i}\right]\left|V_{i}^{n+1}\right| \leq \frac{\Delta \tau}{2} \beta_{i}\left|V_{i-1}^{n+1}\right|} \\
& \quad+\frac{\Delta \tau}{2} w_{i}\left|V_{i+1}^{n+1}\right|+\left(1+\frac{\Delta \tau}{2} \chi_{i}\right)\left|V_{i}^{n}\right| \\
& \quad+\frac{\Delta \tau}{2} \beta_{i}\left|V_{i-1}^{n}\right|+\frac{\Delta \tau}{2}\left|w_{i} V_{i+1}^{n}\right|+\Delta \tau P\left(V^{n+1}\right)_{i i}\left|\Lambda_{i}\right|+\Delta \tau\left|F_{i}^{n}\right| \\
& \leq \frac{\Delta \tau}{2}\left(\beta_{i}+w_{i}\right)\left\|\mathbf{V}^{n+1}\right\|_{\infty}+\left[1+\frac{\Delta \tau}{2}\left(\chi_{i}+\beta_{i}+w_{i}\right)\right]\left\|\mathbf{V}^{n}\right\|_{\infty} \\
& \quad+\Delta \tau P\left(V^{n+1}\right)_{i i}\|\Lambda\|_{\infty}+\Delta \tau\left\|\mathbf{F}^{n}\right\|_{\infty} .
\end{aligned}
$$

Choose $l \in\{1, \ldots, M-1\}$ such that $\left\|\mathbf{V}^{n+1}\right\|_{\infty}=\left|\mathbf{V}_{l}^{n+1}\right|$.

Then, we obtain the following estimate:

Then, (32) becomes

$$
\begin{gathered}
{\left[1-\frac{\Delta \tau}{2} \chi_{l}+\Delta \tau P\left(V^{n+1}\right)_{l l}\right]\left\|V^{n+1}\right\|_{\infty} \leq \frac{\Delta \tau}{2}\left(\beta_{l}+w_{l}\right)\left\|V^{n+1}\right\|_{\infty}} \\
+\left[1+\frac{\Delta \tau}{2}\left(\chi_{l}+\beta_{l}+w_{l}\right)\right]\left\|V^{n}\right\|_{\infty}+\Delta \tau P\left(V^{n+1}\right)_{l l}\|\Lambda\|_{\infty} .
\end{gathered}
$$

$$
\begin{aligned}
\left\|V^{n+1}\right\|_{\infty} & \leq \frac{\max \left(\left\|V^{n}\right\|_{\infty},\|\Lambda\|_{\infty}\right) \times\left[1+(\Delta \tau / 2)\left(\chi_{l}+\beta_{l}+w_{l}\right)+\Delta \tau P\left(V^{n+1}\right)_{l l}\right]}{1+(\Delta \tau / 2)\left(-\chi_{l}-\beta_{l}-w_{l}\right)+\Delta \tau P\left(V^{n+1}\right)_{l l}} \\
& =\frac{\max \left(\left\|V^{n}\right\|_{\infty},\|\Lambda\|_{\infty}\right) \times\left[1-(\Delta \tau / 2) r_{l}+\Delta \tau P\left(V^{n+1}\right)_{l l}\right]}{1+(\Delta \tau / 2) r_{l}+\Delta \tau P\left(V^{n+1}\right)_{l l}} \\
& \leq \max \left(\left\|V^{n}\right\|_{\infty},\|\Lambda\|_{\infty}\right),
\end{aligned}
$$

where $-\chi_{l}-\beta_{l}-w_{l}=r_{l}>0$. Combining (7) and (34) gives

$$
\begin{aligned}
\left\|V^{n+1}\right\|_{\infty} & \leq \max \left(\left\|V^{n}\right\|_{\infty},\|\Lambda\|_{\infty}\right) \leq \cdots \leq \max \left(\left\|V^{0}\right\|_{\infty},\|\Lambda\|_{\infty}\right) \\
& =\|\Lambda\|_{\infty},
\end{aligned}
$$

for all feasible $n$. Hence, the numerical scheme (26) is stable.
The monotonicity of the numerical scheme (26) is given in the following lemma.

Lemma 3. The numerical scheme (26) is unconditionally monotone.

Proof. For $i=0$ or $M$, the lemma is trivially true. When $0<i<M$, it follows from Theorem 1 that the matrices $-(\Delta \tau / 2) \mathbf{S}$ and $\mathbf{I}-(\Delta \tau / 2) \mathbf{S}$ are $M$-matrices, and hence, 
$\left[(\mathbf{I}-\Delta \tau / 2 \mathbf{S}) \mathbf{V}^{n+1}\right]_{i}$ is a strictly increasing function of $\mathbf{V}_{i}^{n+1}$ and nonincreasing function of $\mathbf{V}_{i+1}^{n+1}$ and $\mathbf{V}_{i-1}^{n+1}$, and $\left[(-(\Delta \tau / 2) \mathbf{S}) \mathbf{V}^{n}\right]_{i}$ is also a strictly increasing function of $\mathbf{V}_{i}^{n}$ and nonincreasing function of $\mathbf{V}_{i+1}^{n}$ and $\mathbf{V}_{i-1}^{n}$. On the other hand, it is obvious that $-\mathbf{V}_{i}^{n}$ is a decreasing function of $\mathbf{V}_{i}^{n}$. Finally, the penalty term $-\Delta \tau \mathbf{P}\left(\mathbf{V}^{n+1}\right)_{i i}\left(\Lambda_{i}-\mathbf{V}_{i}^{n+1}\right)$ is a nondecreasing function of $\mathbf{V}_{i}^{n+1}$. Hence, the numerical scheme (26) is unconditionally monotone.

It has been shown in [27] that the solution of the numerical scheme (26) will converge to the viscosity solution as the discretization is consistent, stable, and monotone. Hence, the following theorem follows from Lemmas 1-3.

Theorem 2. The solution of the numerical scheme (26) converges to the viscosity solution of (10) as $h \longrightarrow 0$.

\section{Iterative Algorithm for the Discrete System}

In this section, to solve the nonlinear algebraic system (26) effectively, we propose and analyze an iterative algorithm for (26) at each time step, which is given in Algorithm 1.

Now, we show the convergence of this iterative algorithm for the nonlinear algebraic system (26) in the following theorem.

Theorem 3. At each time step $n$ of the iterative scheme, equation in Algorithm 1 generates a sequence of solutions $\left\{\widehat{\mathbf{V}}^{l}\right\}$, starting from any initial guess $\widehat{\mathbf{V}}^{0}$, that converges monotonically to the solution $\mathbf{V}^{n+1}$ to $(26)$ as $l \longrightarrow \infty$, provided that $h$ is sufficiently small.

Proof. First, we show that the iterate $\left\{\widehat{\mathbf{V}}^{l}\right\}$ is bounded for any $l$. Writing out the equation in Algorithm 1 in component form gives

$$
\begin{aligned}
& {\left[1-\frac{\Delta \tau}{2} \chi_{i}+\Delta \tau P\left(\widehat{V}^{l}\right)_{i i}\right] \widehat{V}_{i}^{l+1}-\frac{\Delta \tau}{2} \beta_{i} \widehat{V}_{i-1}^{l+1}-\frac{\Delta \tau}{2} w_{i} \widehat{V}_{i+1}^{l+1}} \\
& =\left(1+\frac{\Delta \tau}{2} \chi_{i}\right) V_{i}^{n}+\frac{\Delta \tau}{2} \beta_{i} V_{i-1}^{n}+\frac{\Delta \tau}{2} w_{i} V_{i+1}^{n} \\
& +\Delta \tau P\left(\widehat{V}^{l}\right)_{i i} \Lambda_{i}+\Delta \tau F_{i}^{n} .
\end{aligned}
$$

As in the proof of Lemma 2, it follows from $\beta_{i} \geq 0, w_{i} \geq 0$, and $\chi_{i} \leq 0$ that

$$
\begin{aligned}
& {\left[1-\frac{\Delta \tau}{2} \chi_{i}+\Delta \tau P\left(q \widehat{V}^{l}\right)_{i i}\right]\left|\widehat{V}_{i}^{l+1}\right|} \\
& \leq \frac{\Delta \tau}{2} \beta_{i}\left|V_{i-1}^{l+1}\right|+\frac{\Delta \tau}{2} w_{i}\left|V_{i+1}^{l+1}\right|+\left(1+\frac{\Delta \tau}{2} \chi_{i}\right)\left|V_{i}^{n}\right| \\
& \quad+\frac{\Delta \tau}{2} \beta_{i}\left|V_{i-1}^{n}\right|+\frac{\Delta \tau}{2} w_{i}\left|V_{i+1}^{n}\right|+\Delta \tau P\left(V^{l}\right)_{i i}\left|\Lambda_{i}\right|+\Delta \tau\left|F_{i}^{n}\right| \\
& \leq \frac{\Delta \tau}{2}\left(\beta_{i}+w_{i}\right)\left\|\widehat{\mathbf{V}}^{l+1}\right\|_{\infty}+\left[1+\frac{\Delta \tau}{2}\left(\chi_{i}+\beta_{i}+w_{i}\right)\right]\left\|\mathbf{V}^{n}\right\|_{\infty} \\
& \quad+\Delta \tau P\left(\widehat{V}^{l}\right)_{i i}\|\Lambda\|_{\infty}+\Delta \tau\left\|\mathbf{F}^{n}\right\|_{\infty} .
\end{aligned}
$$

Using a similar argument as used in proving Lemma 2, when $h$ is sufficiently small, we have

$$
\left\|\widehat{\mathbf{v}}^{l+1}\right\|_{\infty} \leq\|\Lambda\|_{\infty},
$$

i.e., $\left\|\widehat{\mathbf{V}}^{l}\right\|_{\infty}$ is bounded independently of $l$.

Second, we show that the iterates $\left\{\widehat{\mathbf{V}}^{l}\right\}$ form a nondecreasing sequence. The $l$ th iteration of the equation in Algorithm 1 is given by

$$
\begin{aligned}
& {\left[\mathbf{I}-\frac{\Delta \tau}{2} \mathbf{S}+\Delta \tau \mathbf{P}\left(\widehat{\mathbf{V}}^{l-1}\right)\right] \widehat{\mathbf{V}}^{l}=\left(\mathbf{I}+\frac{\Delta \tau}{2} \mathbf{S}\right) \mathbf{V}^{n}} \\
& +\Delta \tau \mathbf{P}\left(\widehat{\mathbf{V}}^{l-1}\right) \Lambda+\Delta \tau \mathbf{F}^{n},
\end{aligned}
$$

which can be rewritten as follows:

$$
\left(\mathbf{I}-\frac{\Delta \tau}{2} \mathbf{S}\right) \widehat{\mathbf{V}}^{l}=\left(\mathbf{I}+\frac{\Delta \tau}{2} \mathbf{S}\right) \mathbf{V}^{n}+\Delta \tau \mathbf{P}\left(\widehat{\mathbf{V}}^{l-1}\right)\left(\Lambda-\widehat{\mathbf{V}}^{l}\right)+\Delta \tau \mathbf{F}^{n} .
$$
have

Subtracting (40) from the equation in Algorithm 1, we

$$
\begin{aligned}
\left(\mathbf{I}-\frac{\Delta \tau}{2} \mathbf{S}\right)\left(\hat{\mathbf{V}}^{l+1}-\widehat{\mathbf{V}}^{l}\right)= & \Delta \tau\left[\mathbf{P}\left(\widehat{\mathbf{V}}^{l}\right)-\mathbf{P}\left(\widehat{\mathbf{V}}^{l-1}\right)\right] \Lambda \\
& +\Delta \tau\left[\mathbf{P}\left(\widehat{\mathbf{V}}^{l-1}\right) \widehat{\mathbf{V}}^{l}-\mathbf{P}\left(\widehat{\mathbf{V}}^{l}\right) \widehat{\mathbf{V}}^{l+1}\right] .
\end{aligned}
$$

The above scheme can be written as follows:

$$
\begin{aligned}
{[\mathbf{I}} & \left.-\frac{\Delta \tau}{2} \mathbf{S}+\Delta \tau \mathbf{P}\left(\widehat{\mathbf{V}}^{l}\right)\right]\left(\widehat{\mathbf{V}}^{l+1}-\widehat{\mathbf{V}}^{l}\right) \\
& =\Delta \tau\left[\mathbf{P}\left(\hat{\mathbf{V}}^{l}\right)-\mathbf{P}\left(\hat{\mathbf{V}}^{l-1}\right)\right]\left(\Lambda-\hat{\mathbf{V}}^{l}\right) .
\end{aligned}
$$

Noting (42), we examine each component of $\left[\mathbf{P}\left(\widehat{\mathbf{V}}^{l}\right)-\right.$ $\left.\mathbf{P}\left(\widehat{\mathbf{V}}^{l-1}\right)\right]\left(\Lambda-\widehat{\mathbf{V}}^{l}\right)$ in the following two cases.

Case 1. If $\widehat{\mathbf{V}}_{i}^{l}<\Lambda_{i}$, then we have $\mathbf{P}\left(\widehat{\mathbf{V}}^{l}\right)_{i i}=\gamma$, and hence

$$
\left[\mathbf{P}\left(\widehat{\mathbf{V}}^{l}\right)-\mathbf{P}\left(\widehat{\mathbf{V}}^{l-1}\right)\right]\left(\Lambda-\widehat{\mathbf{V}}^{l}\right)=\left[\gamma-\mathbf{P}\left(\widehat{\mathbf{V}}^{l-1}\right)_{i i}\right]\left(\Lambda_{i}-\widehat{\mathbf{V}}_{i}^{l}\right) \geq 0 .
$$

Case 2. If $\widehat{\mathbf{V}}_{i}^{l} \geq \Lambda_{i}$, then we have $\mathbf{P}\left(\widehat{\mathbf{V}}^{l}\right)_{i i}=0$, and hence

$$
\left[\mathbf{P}\left(\hat{\mathbf{V}}^{l}\right)-\mathbf{P}\left(\hat{\mathbf{V}}^{l-1}\right)\right]\left(\Lambda-\hat{\mathbf{V}}^{l}\right)=-\mathbf{P}\left(\hat{\mathbf{V}}^{l-1}\right)_{i i}\left(\Lambda_{i}-\hat{\mathbf{V}}_{i}^{l}\right) \geq 0 .
$$

Thus,

$$
\left[\mathbf{P}\left(\widehat{\mathbf{V}}^{l}\right)-\mathbf{P}\left(\widehat{\mathbf{V}}^{l-1}\right)\right]\left(\Lambda-\widehat{\mathbf{V}}^{l}\right) \geq 0
$$

Combining (42) with (45), we have

$$
\left[\mathbf{I}-\frac{\Delta \tau}{2} \mathbf{S}+\Delta \tau \mathbf{P}\left(\widehat{\mathbf{V}}^{l}\right)\right]\left(\widehat{\mathbf{V}}^{l+1}-\widehat{\mathbf{V}}^{l}\right) \geq 0 .
$$


(1) Let $n=0$.

(2) Set $l=0$ and $\widehat{\mathbf{V}}^{0}=\mathbf{V}^{n}$.

(3) Solve $\left[\mathbf{I}-(\Delta \tau / 2) \mathbf{S}+\Delta \tau \mathbf{P}\left(\widehat{\mathbf{V}}^{l}\right)\right] \widehat{\mathbf{V}}^{l+1}=(\mathbf{I}+(\Delta \tau / 2) \mathbf{S}) \mathbf{V}^{n}+\Delta \tau \mathbf{P}\left(\widehat{\mathbf{V}}^{l}\right) \Lambda+\Delta \tau \mathbf{F}^{n}$

(4) If $\max _{i}\left(\left|\widehat{\mathbf{V}}_{i}^{l+1}-\widehat{\mathbf{V}}_{i}^{l}\right| / \max \left(1,\left|\widehat{\mathbf{V}}_{i}^{l+1}\right|\right)\right)<$ tolerance then stop; otherwise, set $l$ : $=l+1$ and go to Step 3 .

(5)Set $\mathbf{V}^{n+1}=\hat{\mathbf{V}}^{l}, n=n+1$ and go to Step 2 .

Algorithm 1: Iterative algorithm for (26).

TABle 1: The prices of the American put option on a zero-coupon bond with $\theta=0.03$ and $\sigma=0.1$ as $m=n$, where $r_{\max }=2$.

\begin{tabular}{lccccccccc}
\hline & \multicolumn{3}{c}{$r=0.9$} & & \multicolumn{3}{c}{$r=1.0$} & \multicolumn{2}{c}{$r=1.1$} \\
& Value & Error & Order & Value & Error & Order & Value & Error & Order \\
\hline 100 & 54.581754814 & - & - & 56.055386819 & - & - & 57.120900432 & - \\
200 & 54.581756992 & $2.18 e-6$ & - & 56.055388670 & $1.85 e-6$ & - & 57.120901971 & $1.54 e-6$ & - \\
400 & 54.581757538 & $5.46 e-7$ & 2.0 & 56.055389134 & $4.64 e-7$ & 2.0 & 57.120902356 & $3.85 e-7$ & 2.0 \\
800 & 54.581757674 & $1.36 e-7$ & 2.0 & 56.055389250 & $1.16 e-7$ & 2.0 & 57.120902452 & $9.64 e-8$ & 2.0 \\
1600 & 54.581757709 & $3.41 e-8$ & 2.0 & 56.055389279 & $2.90 e-8$ & 2.0 & 57.120902476 & $2.41 e-8$ & 2.0 \\
\hline
\end{tabular}

TABle 2: The prices of the American put option on a zero-coupon bond with $\theta=0.03$ and $\sigma=0.8$ as $m=n$, where $r_{\max }=2$.

\begin{tabular}{lccccccccc}
\hline & \multicolumn{3}{c}{$r=0.9$} & & \multicolumn{3}{c}{$r=1.0$} & & \multicolumn{2}{c}{$r=1.1$} & & & \\
& Value & Error & Order & Value & Error & Order & Value & Error & Order \\
\hline 100 & 36.304526470 & - & - & 39.768020936 & - & - & 42.722357080 & - \\
200 & 36.304527803 & $1.33 e-6$ & - & 39.768022223 & $1.29 e-6$ & - & 42.722358304 & $1.22 e-6$ & - \\
400 & 36.304528136 & $3.33 e-7$ & 2.0 & 39.768022544 & $3.22 e-7$ & 2.0 & 42.722358610 & $3.06 e-7$ & 2.0 \\
800 & 36.304528219 & $8.33 e-8$ & 2.0 & 39.768022624 & $8.04 e-8$ & 2.0 & 42.722358686 & $7.65 e-8$ & 2.0 \\
1600 & 36.304528240 & $2.08 e-8$ & 2.0 & 39.768022645 & $2.01 e-8$ & 2.0 & 42.722358706 & $1.91 e-8$ & 2.0 \\
\hline
\end{tabular}

TABLE 3: The prices of the American put option on a zero-coupon bond with $\theta=0.08$ and $\sigma=0.1$ as $m=n$, where $r_{\text {max }}=2$.

\begin{tabular}{lcccccccccc}
\hline & \multicolumn{3}{c}{$r=0.8$} & & \multicolumn{3}{c}{$r=1.0$} & & \multicolumn{2}{c}{$r=1.5$} \\
& Value & Error & Order & Value & Error & Order & Value & Error & Order \\
\hline 100 & 52.797853729 & - & - & 56.188221908 & - & - & 59.162911163 & - \\
200 & 52.797855702 & $1.97 e-6$ & - & 56.188223472 & $1.56 e-6$ & - & 59.162911739 & $5.76 e-7$ & - \\
400 & 52.797856197 & $4.95 e-7$ & 2.0 & 56.188223863 & $3.91 e-7$ & 2.0 & 59.162911883 & $1.44 e-7$ & 2.0 \\
800 & 52.797856320 & $1.23 e-7$ & 2.0 & 56.188223961 & $9.80 e-8$ & 2.0 & 59.162911919 & $3.60 e-8$ & 2.0 \\
1600 & 52.797856351 & $3.10 e-8$ & 2.0 & 56.188223985 & $2.40 e-8$ & 2.0 & 59.162911928 & $9.00 e-9$ & 2.0 \\
\hline
\end{tabular}

TABle 4: The prices of the American put option on a zero-coupon bond with $\theta=0.08$ and $\sigma=0.3$ as $m=n$, where $r_{\max }=2$.

\begin{tabular}{|c|c|c|c|c|c|c|c|c|c|}
\hline \multirow{2}{*}{$m$} & \multicolumn{3}{|c|}{$r=0.8$} & \multicolumn{3}{|c|}{$r=1.0$} & \multicolumn{3}{|c|}{$r=1.5$} \\
\hline & Value & Error & Order & Value & Error & Order & Value & Error & Order \\
\hline 100 & 49.640003215 & - & - & 54.020836795 & - & - & 58.432510559 & - & - \\
\hline 200 & 49.640004432 & $1.22 e-6$ & - & 54.020837757 & $9.62 e-7$ & - & 58.432510962 & $4.03 e-7$ & - \\
\hline 400 & 49.640004736 & $3.04 e-7$ & 2.0 & 54.020837998 & $2.41 e-7$ & 2.0 & 58.432511062 & $1.00 e-7$ & 2.0 \\
\hline 800 & 49.640004812 & $7.60 e-8$ & 2.0 & 54.020838058 & $6.00 e-8$ & 2.0 & 58.432511087 & $2.50 e-8$ & 2.0 \\
\hline 1600 & 49.640004831 & $1.90 e-8$ & 2.0 & 54.020838073 & $1.50 e-8$ & 2.0 & 58.432511093 & $6.00 e-9$ & 2.0 \\
\hline
\end{tabular}

TABle 5: The prices of the American put option on a zero-coupon bond with $\theta=0.08$ and $\sigma=0.5$ as $m=n$, where $r_{\max }=2$.

\begin{tabular}{lccccccccc}
\hline & \multicolumn{3}{c}{$r=0.8$} & & \multicolumn{3}{c}{$r=1.0$} & & \multicolumn{2}{c}{$r=1.5$} & & & \\
& Value & Error & Order & Value & Error & Order & Value & Error & Order \\
\hline 100 & 43.763440286 & - & - & 49.554320053 & - & - & 56.486342717 & - \\
200 & 43.763441674 & $1.39 e-6$ & - & 49.554321245 & $1.19 e-6$ & - & 56.486343354 & $6.37 e-7$ & - \\
400 & 43.763442021 & $3.47 e-7$ & 2.0 & 49.554321543 & $2.98 e-7$ & 2.0 & 56.486343513 & $1.59 e-7$ & 2.0 \\
800 & 43.763442108 & $8.70 e-8$ & 2.0 & 49.554321617 & $7.40 e-8$ & 2.0 & 56.486343553 & $4.00 e-8$ & 2.0 \\
1600 & 43.763442130 & $2.20 e-8$ & 2.0 & 49.554321636 & $1.90 e-8$ & 2.0 & 56.486343563 & $1.00 e-8$ & 2.0 \\
\hline
\end{tabular}


TABle 6: The prices of the American put option on a zero-coupon bond with $\theta=0.08$ and $\sigma=0.8$ as $m=n$, where $r_{\max }=2$.

\begin{tabular}{lccccccccc}
\hline & \multicolumn{3}{c}{$r=0.8$} & & \multicolumn{3}{c}{$r=1.0$} & & \multicolumn{2}{c}{$r=1.5$} & & & \\
& Value & Error & Order & Value & Error & Order & Value & Error & Order \\
\hline 100 & 32.874384467 & - & - & 40.226739920 & - & - & 50.994927357 & - \\
200 & 32.874385679 & $1.21 e-6$ & - & 40.226741134 & $1.21 e-6$ & - & 50.994928235 & $8.78 e-7$ & - \\
400 & 32.874385984 & $3.05 e-7$ & 2.0 & 40.226741437 & $3.03 e-7$ & 2.0 & 50.994928455 & $2.20 e-7$ & 2.0 \\
800 & 32.874386061 & $7.70 e-8$ & 2.0 & 40.226741513 & $7.60 e-8$ & 2.0 & 50.994928510 & $5.50 e-8$ & 2.0 \\
1600 & 32.874386080 & $1.90 e-8$ & 2.0 & 40.226741532 & $1.90 e-8$ & 2.0 & 50.994928524 & $1.40 e-8$ & 2.0 \\
\hline
\end{tabular}

TABLE 7: The prices of the American put option on a zero-coupon bond with $\theta=0.08$ and $\sigma=0.1$ as $m=n$, where $r_{\max }=3$.

\begin{tabular}{|c|c|c|c|c|c|c|c|c|c|}
\hline \multirow{2}{*}{$m$} & \multicolumn{3}{|c|}{$r=0.9$} & \multicolumn{3}{|c|}{$r=1.1$} & \multicolumn{3}{|c|}{$r=1.3$} \\
\hline & Value & Error & Order & Value & Error & Order & Value & Error & Order \\
\hline 150 & 54.765185055 & - & - & 57.217095894 & - & - & 58.497251824 & - & - \\
\hline 300 & 54.765186849 & $1.79 e-6$ & - & 57.217097218 & $1.32 e-6$ & - & 58.497252719 & $8.95 e-7$ & - \\
\hline 600 & 54.765187299 & $4.49 e-7$ & 2.0 & 57.217097549 & $3.31 e-7$ & 2.0 & 58.497252943 & $2.24 e-7$ & 2.0 \\
\hline 1200 & 54.765187411 & $1.12 e-7$ & 2.0 & 57.217097632 & $8.29 e-8$ & 2.0 & 58.497252999 & $5.61 e-8$ & 2.0 \\
\hline 2400 & 54.765187439 & $2.81 e-8$ & 2.0 & 57.217097653 & $2.07 e-8$ & 2.0 & 58.497253013 & $1.40 e-9$ & 2.0 \\
\hline
\end{tabular}

TABLE 8: The prices of the American put option on a zero-coupon bond with $\theta=0.08$ and $\sigma=0.3$ as $m=n$, where $r_{\max }=3$.

\begin{tabular}{lccccccccc}
\hline \multirow{2}{*}{} & \multicolumn{3}{c}{$r=0.9$} & \multicolumn{3}{c}{$r=1.1$} & \multicolumn{3}{c}{$r=1.3$} \\
& Value & Error & Order & Value & Error & Order & Value & Error & Order \\
\hline 150 & 52.133287029 & - & - & 55.449461279 & - & - & 57.347886873 & - \\
300 & 52.133288126 & $1.10 e-6$ & - & 55.449462107 & $8.28 e-7$ & - & 57.347887461 & $5.88 e-7$ & - \\
600 & 52.133288400 & $2.74 e-7$ & 2.0 & 55.449462314 & $2.07 e-7$ & 2.0 & 57.347887608 & $1.47 e-7$ & 2.0 \\
1200 & 52.133288468 & $6.85 e-8$ & 2.0 & 55.449462365 & $5.17 e-8$ & 2.0 & 57.347887645 & $3.68 e-8$ & 2.0 \\
2400 & 52.133288485 & $1.71 e-8$ & 2.0 & 55.449462378 & $1.29 e-8$ & 2.0 & 57.347887654 & $9.19 e-9$ & 2.0 \\
\hline
\end{tabular}

TABle 9: The prices of the American put option on a zero-coupon bond with $\theta=0.08$ and $\sigma=0.5$ as $m=n$, where $r_{\max }=3$.

\begin{tabular}{|c|c|c|c|c|c|c|c|c|c|}
\hline \multirow{2}{*}{$m$} & \multicolumn{3}{|c|}{$r=0.9$} & \multicolumn{3}{|c|}{$r=1.1$} & \multicolumn{3}{|c|}{$r=1.3$} \\
\hline & Value & Error & Order & Value & Error & Order & Value & Error & Order \\
\hline 150 & 46.979531081 & - & - & 51.615448216 & - & - & 54.585318188 & - & - \\
\hline 300 & 46.979532382 & $1.30 e-6$ & - & 51.615449290 & $1.07 e-6$ & - & 54.585319030 & $8.41 e-7$ & - \\
\hline 600 & 46.979532707 & $3.25 e-7$ & 2.0 & 51.615449558 & $2.68 e-7$ & 2.0 & 54.585319240 & $2.10 e-7$ & 2.0 \\
\hline 1200 & 46.979532788 & $8.13 e-8$ & 2.0 & 51.615449626 & $6.71 e-8$ & 2.0 & 54.585319292 & $5.26 e-8$ & 2.0 \\
\hline 2400 & 46.979532809 & $2.03 e-8$ & 2.0 & 51.615449642 & $1.68 e-8$ & 2.0 & 54.585319306 & $1.31 e-8$ & 2.0 \\
\hline
\end{tabular}

TABLE 10: The prices of the American put option on a zero-coupon bond with $\theta=0.08$ and $\sigma=0.8$ as $m=n$, where $r_{\max }=3$.

\begin{tabular}{|c|c|c|c|c|c|c|c|c|c|}
\hline \multirow{2}{*}{$m$} & \multicolumn{3}{|c|}{$r=0.9$} & \multicolumn{3}{|c|}{$r=1.1$} & \multicolumn{3}{|c|}{$r=1.3$} \\
\hline & Value & Error & Order & Value & Error & Order & Value & Error & Order \\
\hline 150 & 36.842142692 & - & - & 43.113757292 & - & - & 47.676423742 & - & - \\
\hline 300 & 36.842143940 & $1.25 e-6$ & - & 43.113758453 & $1.16 e-6$ & - & 47.676424768 & $1.03 e-6$ & - \\
\hline 600 & 36.842144252 & $3.12 e-7$ & 2.0 & 43.113758743 & $2.90 e-7$ & 2.0 & 47.676425025 & $2.57 e-7$ & 2.0 \\
\hline 1200 & 36.842144330 & $7.80 e-8$ & 2.0 & 43.113758816 & $7.26 e-8$ & 2.0 & 47.676425089 & $6.42 e-8$ & 2.0 \\
\hline 2400 & 36.842144349 & $1.95 e-8$ & 2.0 & 43.113758834 & $1.81 e-8$ & 2.0 & 47.676425105 & $1.60 e-8$ & 2.0 \\
\hline
\end{tabular}




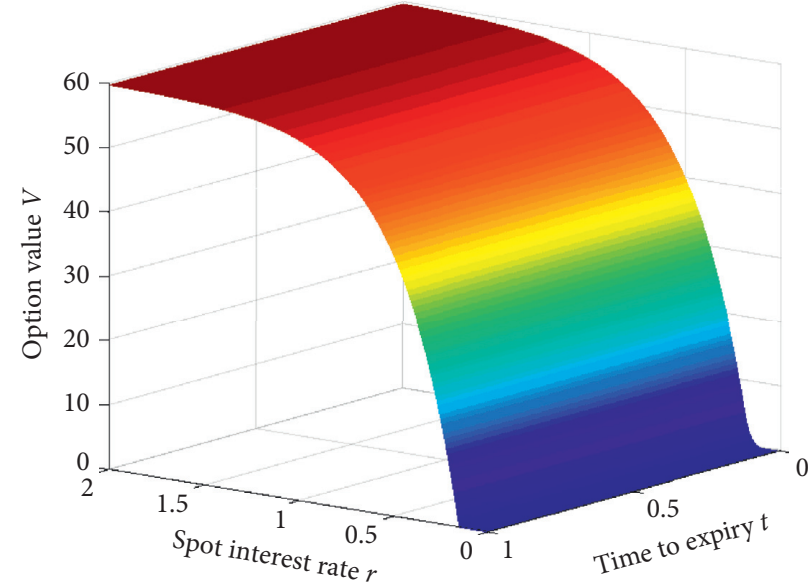

(a)

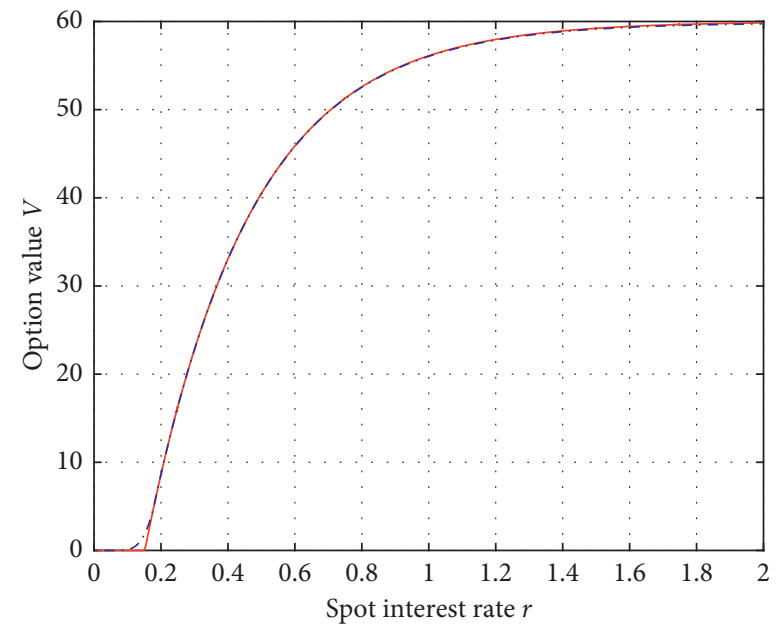

$\begin{array}{ll}\text { - Payoff } \\ - & \theta=0.03, \sigma=0.1\end{array}$

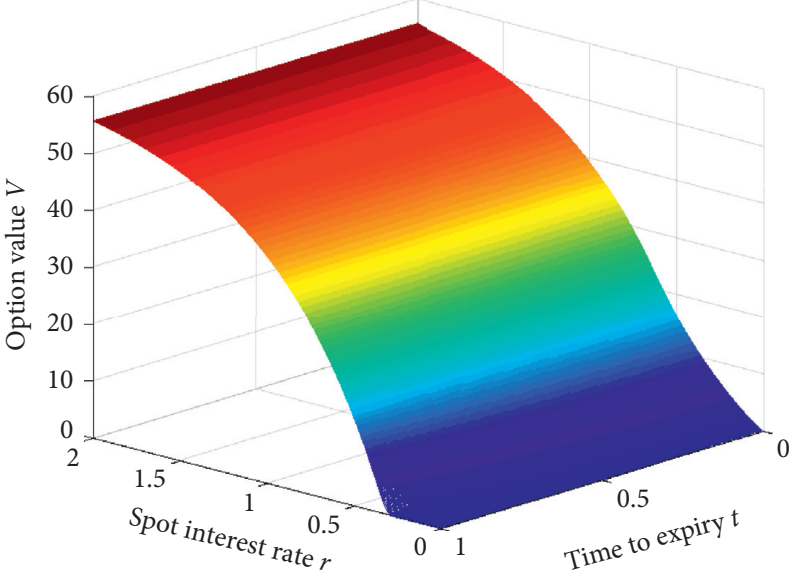

(b)

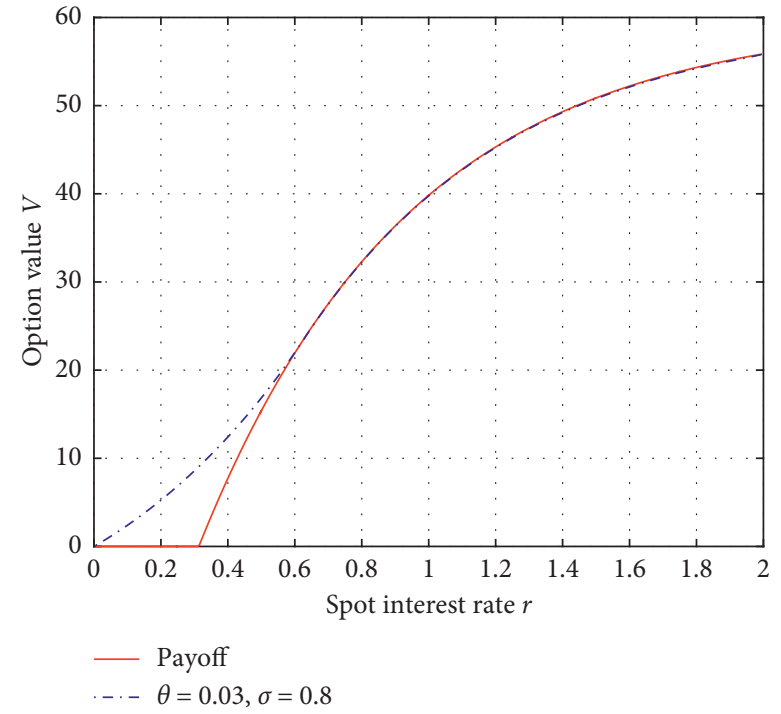

(d)

Figure 1: The American bond option value surface and the option value at $\tau=T$ based on $(m, n)=(400,400)$, where $r_{\max }=2$. (a) $\theta=0.03$, $\sigma=0.1$; (b) $\theta=0.03, \sigma=0.8$; (c) $\theta=0.03, \sigma=0.1$; (d) $\theta=0.03, \sigma=0.8$.

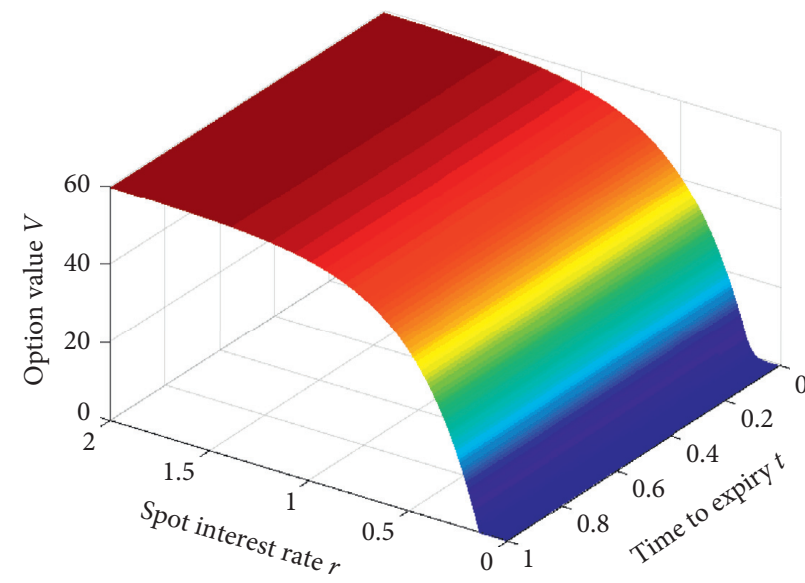

(a)

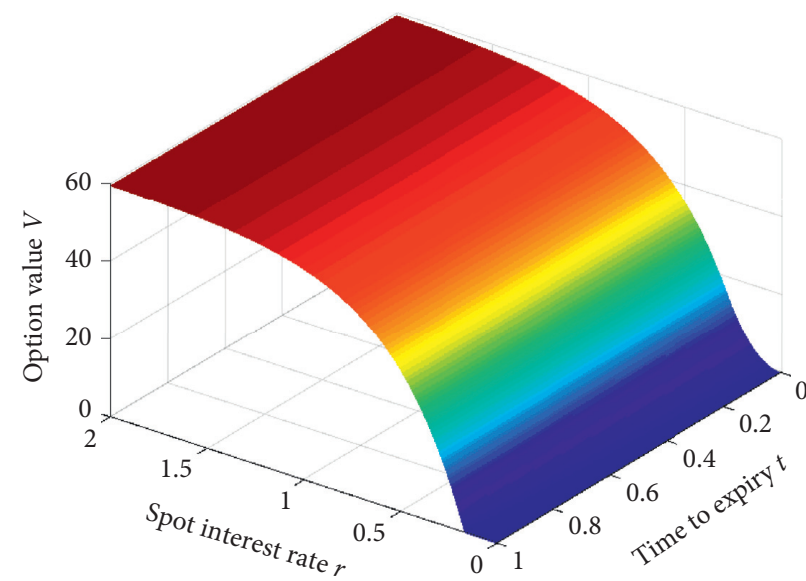

(b)

FIgUre 2: Continued. 


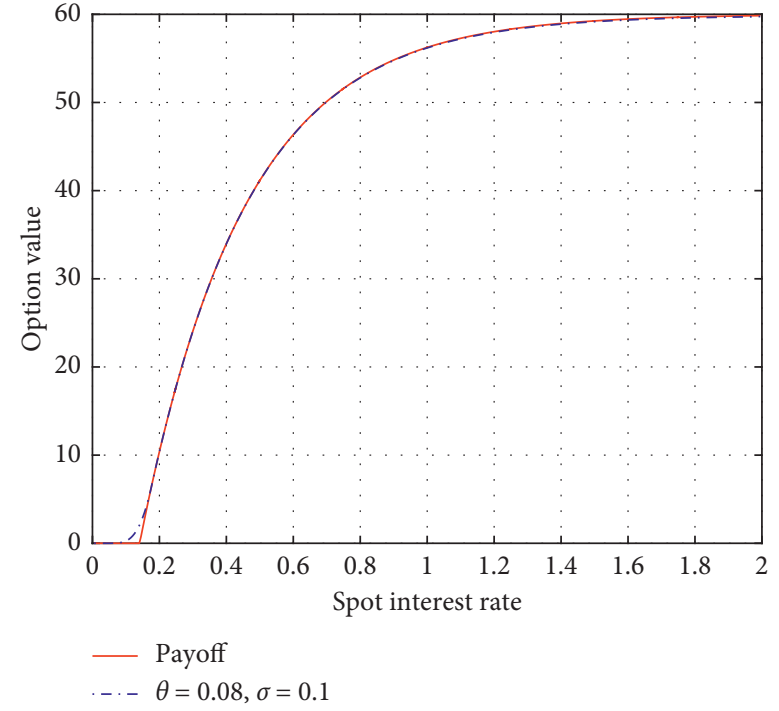

(c)

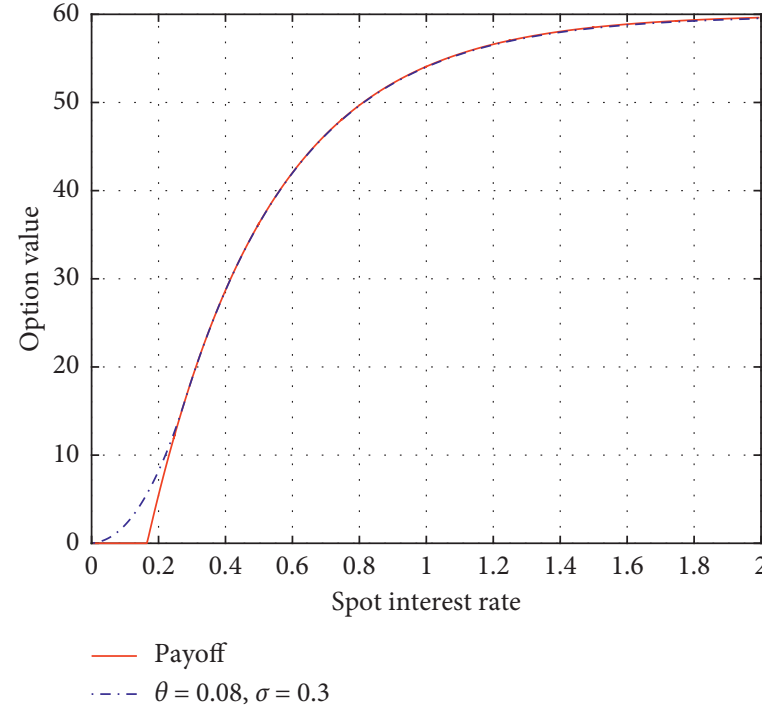

(d)

Figure 2: The American bond option value surface and the option value at $\tau=T$ based on $(m, n)=(400,400)$, where $r_{\max }=2$. (a) $\theta=0.08$, $\sigma=0.1$; (b) $\theta=0.08, \sigma=0.3$; (c) $\theta=0.08, \sigma=0.1$; (d) $\theta=0.08, \sigma=0.3$.

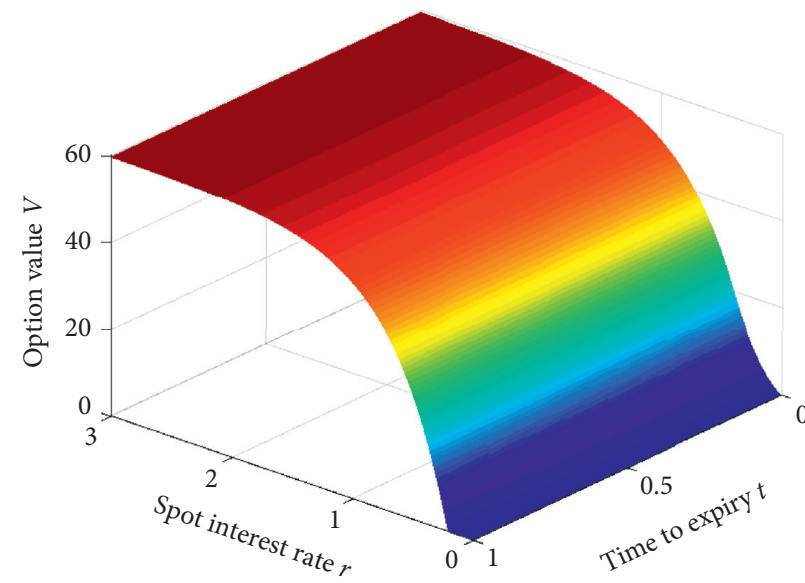

(a)

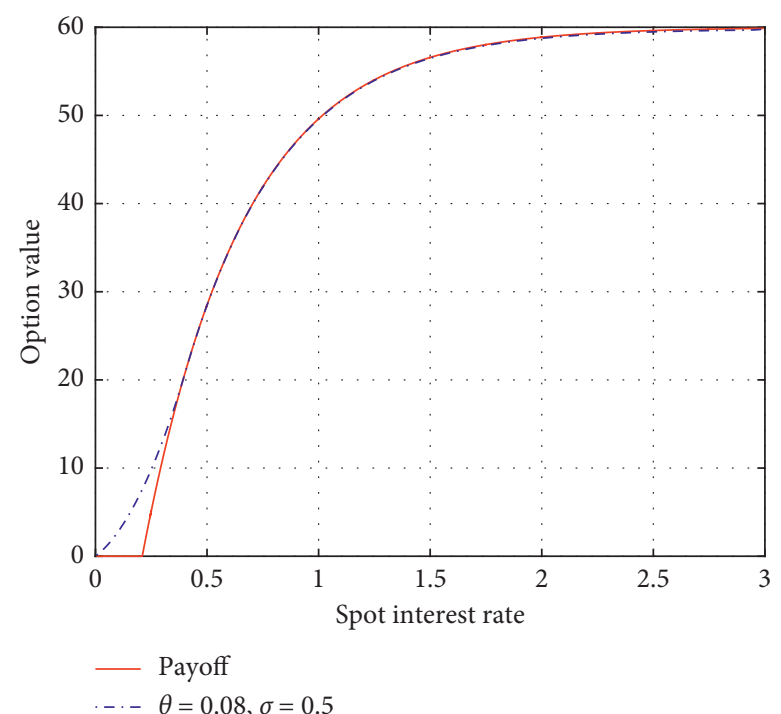

(c)

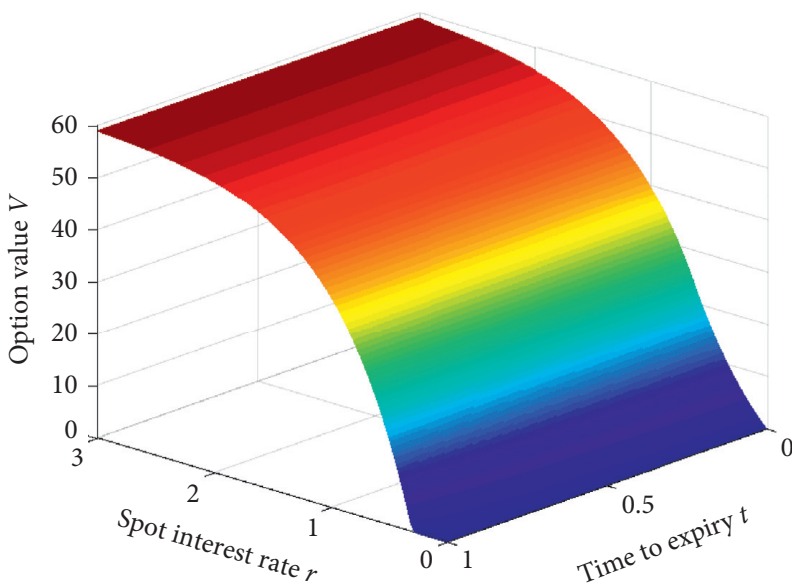

(b)

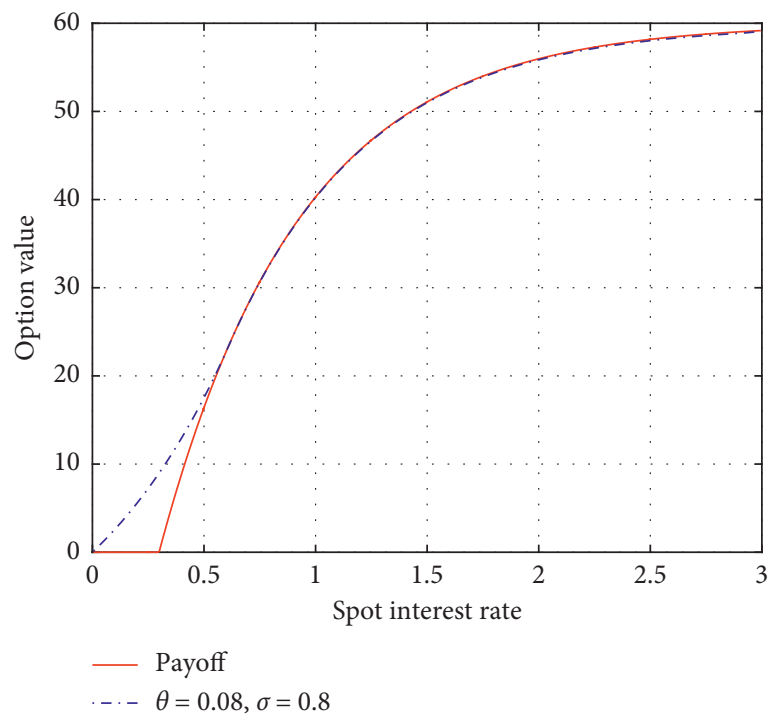

(d)

Figure 3: The American bond option value surface and the option value at $\tau=T$ based on $(m, n)=(600,600)$, where $r_{\max }=3$. (a) $\theta=0.08$, $\sigma=0.5$; (b) $\theta=0.08, \sigma=0.8$; (c) $\theta=0.08, \sigma=0.5$; (d) $\theta=0.08, \sigma=0.8$. 
Further, from Theorem 1, we know that $\mathbf{I}-(\Delta \tau / 2) \mathbf{S}+$ $\Delta \tau \mathbf{P}\left(\widehat{\mathbf{V}}^{l}\right)$ is an $M$-matrix, so the inequality (46) implies

$$
\widehat{\mathbf{V}}^{l+1}-\widehat{\mathbf{V}}^{l} \geq 0
$$

by the discrete maximum principle, and hence, the iterates $\left\{\widehat{\mathbf{V}}^{l}\right\}$ form a nondecreasing sequence.

To summarize, we have shown that the iterates $\left\{\widehat{\mathbf{V}}^{l}\right\}$ form a nondecreasing sequence which is bounded from above. Hence, the sequence converges to the solution of numerical scheme (26).

As for the uniqueness, suppose we have two solutions to the equation in Algorithm $1, \mathbf{B}_{1}$ and $\mathbf{B}_{2}$. Then

$$
\left[\mathbf{I}-\frac{\Delta \tau}{2} \mathbf{S}+\Delta \tau \mathbf{P}\left(\mathbf{B}_{1}\right)\right] \mathbf{B}_{1}=\left(\mathbf{I}+\frac{\Delta \tau}{2} \mathbf{S}\right) \mathbf{V}^{n}+\Delta \tau \mathbf{P}\left(\mathbf{B}_{1}\right) \Lambda+\Delta \tau \mathbf{F}^{n}
$$

$\left[\mathbf{I}-\frac{\Delta \tau}{2} \mathbf{S}+\Delta \tau \mathbf{P}\left(\mathbf{B}_{2}\right)\right] \mathbf{B}_{2}=\left(\mathbf{I}+\frac{\Delta \tau}{2} \mathbf{S}\right) \mathbf{V}^{n}+\Delta \tau \mathbf{P}\left(\mathbf{B}_{2}\right) \Lambda+\Delta \tau \mathbf{F}^{n}$

Some manipulation of (48a) and (48b) results in

$$
\left[\mathbf{I}-\frac{\Delta \tau}{2} \mathbf{S}+\Delta \tau \mathbf{P}\left(\mathbf{B}_{2}\right)\right]\left(\mathbf{B}_{1}-\mathbf{B}_{2}\right)=\Delta \tau\left[\mathbf{P}\left(\mathbf{B}_{1}\right)-\mathbf{P}\left(\mathbf{B}_{2}\right)\right]\left(\Lambda-\mathbf{B}_{1}\right) .
$$
obtain

Using a similar argument as used in proving (46), we

$$
\left[\mathbf{P}\left(\mathbf{B}_{1}\right)-\mathbf{P}\left(\mathbf{B}_{2}\right)\right]\left(\Lambda-\mathbf{B}_{1}\right) \geq 0 .
$$

Since $\mathbf{I}-(\Delta \tau / 2) \mathbf{S}+\Delta \tau \mathbf{P}\left(\mathbf{B}_{2}\right)$ is an $M$-matrix, it follows from (49) and (50) that

$$
\mathbf{B}_{1} \geq \mathbf{B}_{2} \text {. }
$$

By symmetry, we also have

$$
\mathbf{B}_{2} \geq \mathbf{B}_{1} \text {, }
$$

and hence $\mathbf{B}_{1}=\mathbf{B}_{2}$. This completes the proof.

Remark 4. From Theorems 2 and 3, we know that the solution resulting from Algorithm 1 converges to the correct solution of (10), i.e., the unique viscosity solution or financially relevant solution.

\section{Numerical Experiments}

In this section, we present some numerical results to demonstrate the performance and convergence of the new numerical scheme. In the sequel, "error" refers to the difference between successive numerical solutions following mesh refinements, i.e.,

$$
\text { error }=\left|V_{\Delta r}^{\Delta \tau}-V_{\Delta r / 2}^{\Delta \tau / 2}\right|
$$

where $V_{\Delta r}^{\Delta \tau}$ denotes the computed solution on the spatial mesh $\Delta r$ and time mesh size $\Delta \tau$, and "order" is $\log _{2}$ of the ratio of successive changes in option price as the grid is refined. Further, the penalty parameter $\gamma$ and the tolerance are chosen to be $10^{3}$ and $10^{-8}$ in Algorithm 1, respectively. All codes were carried out in Matlab R2016a with $32.00 \mathrm{~GB}$ RAM and $3.20 \mathrm{GHz}$ processor.

The assumed parameters for American put options on a zero-coupon bond under the CIR model are (cf. [11]) as follows:

$$
\begin{aligned}
\kappa & =0.1, \\
\theta & =0.03 \text { or } 0.08, \\
\sigma & =0.1,0.3,0.5 \text { or } 0.8, \\
\zeta & =0, \\
E & =100, \\
K & =60, \\
T & =1, \\
s & =5, \\
r_{\max } & =2 \text { or } 3,
\end{aligned}
$$

and then the grid is defined as consecutive uniform partitions of the domain $\left[0, r_{\max }\right] \times[0, T]$ with $m$ and $n$ being the space and time steps, respectively.

Tables 1-2 list the prices of the American put option on a zero-coupon bond under the CIR model at $\theta=0.03$ and $r=$ $0.9,1.0,1.1$ for different values of $\sigma$, where $r_{\max }=2$. Tables 3-6 list the prices at $\theta=0.08$ and $r=0.8,1.0,1.5$ for different values of $\sigma$, where $r_{\max }=2$. And Tables $7-10$ list the prices at $\theta=0.08$ and $r=0.9,1.1,1.3$ for different values of $\sigma$, where $r_{\max }=3$. From the results in these tables, we can observe that the accuracy of the numerical solutions of the American put options on a zero-coupon bond improves as the discretization grid is refined, and the order of convergence of the Crank-Nicolson scheme is roughly 2. This is consistent with the properties of the Crank-Nicolson scheme. Some authors have also used this approach when an analytic solution is not available [25, 31, 32]. Further, to visualize the numerical solutions, we plot the values of the American put options on a zero-coupon bond with different values of $\sigma$ in Figures 1-3. From these figures, we can see that the numerical solutions are qualitatively excellent and the plots also indicate that our numerical scheme is stable.

\section{Conclusion}

We developed and analysed a Crank-Nicolson fitted finite volume method for pricing American options on a zerocoupon bond model. Based on a penalty approach, we first approximate the original problem by a nonlinear PDE. After that, a novel fitted finite volume method for the spatial discretization of the nonlinear penalized PDE system is coupled with the Crank-Nicolson time-stepping scheme. It is shown that this scheme is consistent, stable, and monotone, and hence, the convergence was guaranteed. To solve the nonlinear algebraic system effectively, an iterative algorithm was designed and its convergence was proved. Numerical experiments were performed to demonstrate the convergence, efficiency, and robustness of the new numerical method. Compared with the fully implicit time- 
stepping schemes proposed by Zhang et al. in [11, 14], the Crank-Nicolson scheme here delivers more accurate approximate solutions.

\section{Data Availability}

The data used to support the findings of this study are available from the corresponding author upon request.

\section{Conflicts of Interest}

The authors declare that they have no conflicts of interest.

\section{Acknowledgments}

This work was supported by the National Natural Science Foundation of China (no. 61463002), the Science and Technology Program of Yunnan Province of China (2019FH001-079), and the Yunnan Provincial Department of Education Science Research Fund Project (no. 2019J0396).

\section{References}

[1] G. H. Deng, "Pricing American put option on zero-coupon bond in a jump-extended CIR model," Communications in Nonlinear Science and Numerical Simulation, vol. 22, no. 1-3, pp. 186-196, 2015.

[2] Y. K. Kwok, Mathematical Models of Financial Derivatives, Springer, Berlin, Germany, 1998.

[3] H. Yang, "Calibration of the extended CIR model," SIAM Journal on Applied Mathematics, vol. 66, no. 2, pp. 721-735, 2006.

[4] S. J. Li and S. H. Li, "Pricing American interest rate option on zero-coupon bond numerically," Applied Mathematics and Computation, vol. 175, no. 1, pp. 834-850, 2006.

[5] W. Allegretto, Y. Lin, and H. Yang, "Numerical pricing of American put options on zero-coupon bonds," Applied Numerical Mathematics, vol. 46, no. 2, pp. 113-134, 2003.

[6] T. Chernogorova and R. Valkov, "Analysis of a finite volume element method for a degenerate parabolic equation in the zero-coupon bond pricing," Computational and Applied Mathematics, vol. 34, no. 2, pp. 619-646, 2015.

[7] P. Edson and C. P. Kailash, "A robust spectral method for pricing of American put options on zero-coupon bonds," East Asian Journal on Applied Mathematics, vol. 8, no. 1, pp. 126-138, 2017.

[8] A. Falcó, L. Navarro, and C. Vázquez, "A direct LU solver for pricing American bond options under Hull-White model," Journal of Computational and Applied Mathematics, vol. 309, pp. $442-455,2017$.

[9] A. D. Holmes and H. Yang, "A front-fixing finite element method for the valuation of American put options on zerocoupon bonds," International Journal of Numerical Analysis and Modeling, vol. 9, no. 4, pp. 777-792, 2012.

[10] H. T. Yang, "American put options on zero-coupon bonds and a parabolic free boundary problem," International Journal of Numerical Analysis and Modeling, vol. 1, no. 2, pp. 203-215, 2004.

[11] K. Zhang and S. Wang, "Pricing American bond options using a penalty method," Automatica, vol. 48, no. 3, pp. 472-479, 2012.
[12] K. Zhang, "Applying a power penalty method to numerically pricing American bond options," Journal of Optimization Theory and Applications, vol. 154, no. 1, pp. 278-291, 2012.

[13] H. J. Zhou, K. F. C. Yiu, and L. K. Li, "Evaluating American put options on zero-coupon bonds by a penalty method," Journal of Computational and Applied Mathematics, vol. 235, no. 13, pp. 3921-3931, 2011.

[14] K. Zhang and X. Q. Yang, "Pricing European options on zerocoupon bonds with a fitted finite volume method," International Journal of Numerical Analysis and Modeling, vol. 14, no. 3, pp. 405-418, 2017.

[15] Y. Tian, "A simplified binomial approach to the pricing of interest-rate contingent claims," International Journal of Financial Engineering, vol. 1, no. 1, pp. 14-37, 1992.

[16] S. Wang, "A novel fitted finite volume method for the BlackScholes equation governing option pricing," IMA Journal of Numerical Analysis, vol. 24, no. 4, pp. 699-720, 2004.

[17] S. Chang, J. Wang, and X. Wang, "A fitted finite volume method for real option valuation of risks in climate change," Computers \& Mathematics with Applications, vol. 70, no. 5, pp. 1198-1219, 2015.

[18] C.-S. Huang, C.-H. Hung, and S. Wang, "A fitted finite volume method for the valuation of options on assets with stochastic volatilities," Computing, vol. 77, no. 3, pp. 297-320, 2006.

[19] K. Zhang and S. Wang, "A computational scheme for uncertain volatility model in option pricing," Applied Numerical Mathematics, vol. 59, no. 8, pp. 1754-1767, 2009.

[20] K. Zhang, K. L. Teo, and M. Swartz, "A robust numerical scheme for pricing American options under regime switching based on penalty method," Computational Economics, vol. 43, no. 4, pp. 463-483, 2014.

[21] K. Zhang and X. Yang, "Power penalty approach to American options pricing under regime switching," Journal of Optimization Theory and Applications, vol. 179, no. 1, pp. 311-331, 2018.

[22] T. Zhang, "Two-grid characteristic finite volume methods for nonlinear parabolic problem," Journal of Computational Mathematics, vol. 31, no. 5, pp. 470-487, 2013.

[23] T. Zhang, H. Zhong, and J. Zhao, "A full discrete two-grid finite-volume method for a nonlinear parabolic problem," International Journal of Computer Mathematics, vol. 88, no. 8, pp. 1644-1663, 2011.

[24] J. C. Cox, J. E. Ingersoll, and S. A. Ross, "A theory of the term structure of interest rates," Econometrica, vol. 53, no. 2, pp. 385-407, 1985.

[25] P. A. Forsyth and K. R. Vetzal, "Quadratic convergence for valuing American options using a penalty method," SIAM Journal on Scientific Computing, vol. 23, no. 6, pp. 2095-2122, 2002.

[26] A. L. Amadori, "Nonlinear integro-differential evolution problems arising in option pricing: a viscosity solution approach," Differential Integral Equations, vol. 16, no. 7, pp. 787-811, 2003.

[27] G. Barles, "Convergence of numerical schemes for degenerate parabolic equations arising in finance," in Numerical Methods in Finance, L. C. G. Rogers and D. Talay, Eds., pp. 1-21, Cambridge University Press, Cambridge, UK, 1997.

[28] W. Li and S. Wang, "Penalty approach to the HJB equation arising in European stock option pricing with proportional transaction costs," Journal of Optimization Theory and Applications, vol. 143, no. 2, pp. 279-293, 2009.

[29] S. Wang, X. Q. Yang, and K. L. Teo, "Power penalty method for a linear complementarity problem arising from American 
option valuation," Journal of Optimization Theory and Applications, vol. 129, no. 2, pp. 227-254, 2006.

[30] R. S. Varga, Matrix Iterative Analysis, Springer, Dordrecht, The Netherlands, Second edition, 1999.

[31] K. Kazmi, "An IMEX predictor-corrector method for pricing options under regime-switching jump-diffusion models," International Journal of Computer Mathematics, vol. 95, no. 3, pp. 1-21, 2018.

[32] K. Kazmi, A. Q. M. Khaliq, and S. Alrabeei, "Solving complex PIDE systems for pricing American option under multi-state regime switching jump-diffusion model," Computers \& Mathematics with Applications, vol. 75, no. 8, pp. 2989-3001, 2018. 